\title{
Wireless power takes control
}

In a world of mobile devices and electric vehicles, wireless energy delivery is of ever-increasing value.

W ireless power transfer is not a new idea - demonstrations of such technology can be traced back over a century, to the work of Nikola Tesla ${ }^{1}$ - but the approach has received renewed attention in recent years due to the increasing ubiquity of mobile electronic devices. Commercial systems are now available, including those for charging mobile phones and tablets, as well those for charging electric vehicles. Novel applications in implantable medical devices $^{2}$, untethered robotics ${ }^{3}$ and wearable sensors $^{4}$ are also being actively explored. Nevertheless, power delivery remains, in general, a relatively wired experience, at least compared to the wireless nature of data connectivity.

A variety of methods have been developed to transfer power wirelessly. The most common of these is via inductive coupling and, in particular, through the use of two magnetically coupled resonant coils ${ }^{5}$. This approach does have restrictions in terms of range, which is typically up to around one metre and is dependent on the size of the resonator used ${ }^{6}$. It also is usually limited to the charging of static devices, though techniques to potentially deliver power transfer to moving devices - or even vehicles - have been reported ${ }^{7}$. But wireless power can also be used for more than just charging. In an Article in this issue of Nature Electronics, Feng Zhu, Oliver Schmidt and colleagues show that wireless power can be used to drive and control a flexible microelectronic system.

With the help of microelectronic fabrication techniques, the researchers - who are based at Chemnitz University of Technology, Leibniz IFW Dresden, Changchun Institute of Applied Chemistry and Dresden University of Technology - built microbots composed of two tubeshaped catalytic micro-engines supported on a flat polymeric structure. A square coil is integrated into the structure, allowing energy to be wirelessly transferred to the devices via inductive coupling. The transferred energy can heat wires embedded in the catalytic engines, increasing the rate

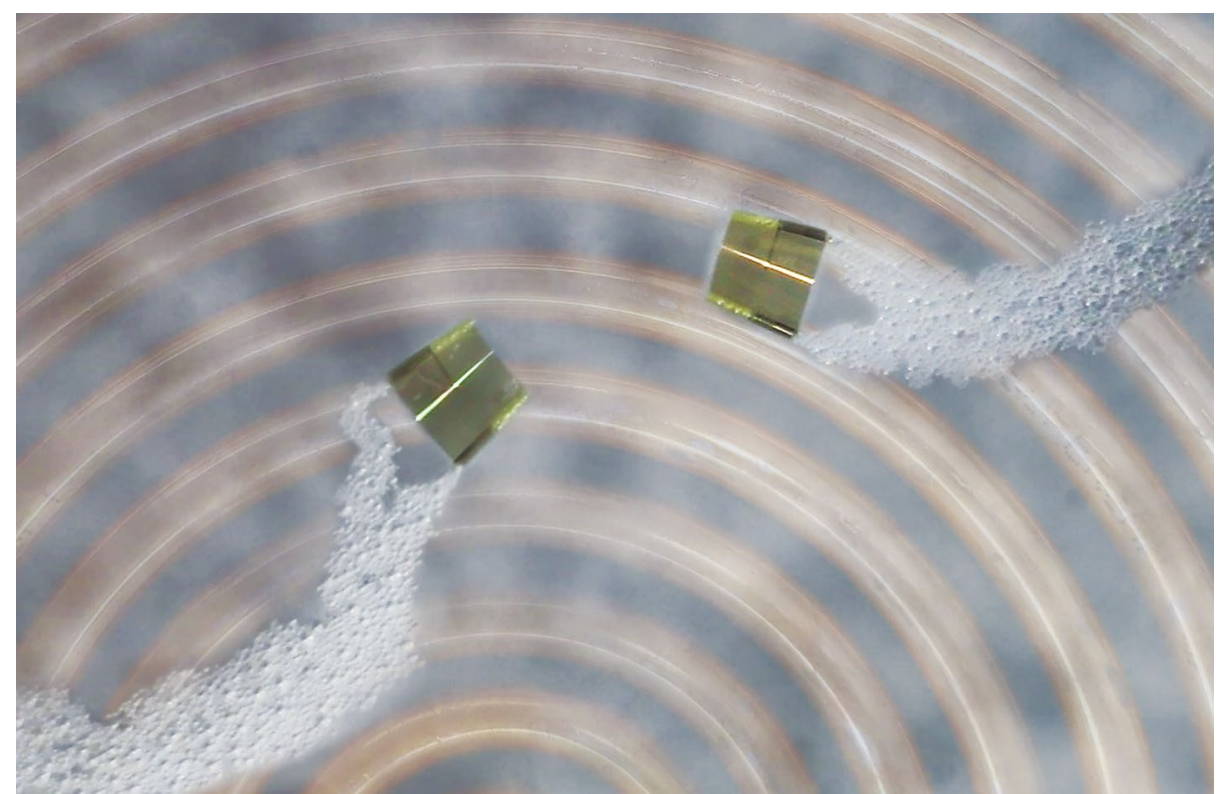

Optical image of the microbots developed by Feng Zhu, Oliver Schmidt and colleagues, which can be driven and controlled with wireless power. Credit: Oliver G. Schmidt, Feng Zhu, Vineeth K. Bandari, Yu Hong; TU Chemnitz, Chemnitz

at which oxygen microbubbles - used by the miniature jet engines to provide propulsion - are generated. And by switching this heating on and off, the microbots can be steered remotely.

Zhu, Schmidt and colleagues also demonstrate that other electronic devices, such as light-emitting diodes, can be integrated into the structures and controlled by wireless power. Furthermore, the microbots can be upgraded with polymeric micro-arms that swell and shrink in response to local temperature changes, which are manipulated through inductive heating. These arms can be used to perform basic grasp and release tasks.

There is interest in using such robots for biomedical applications, including drug delivery and biosensing. As Changhao Xu and Wei Gao at the California Institute of Technology explain in an accompanying News \& Views article, precise motion control remains an issue in the development of these devices. The approach of $\mathrm{Zhu}$, Schmidt and colleagues highlights the potential of wireless power to provide such capabilities. However, modifications to the technique would be required before biomedical microbots could become a reality. For a start, the jet engines are fuelled by hydrogen peroxide (which is decomposed by the catalyst into oxygen microbubbles and water) and thus finding an alternative fuel will be essential.

Published online: 20 March 2020 https://doi.org/10.1038/s41928-020-0398-8

References

1. Tesla, N. Apparatus for transmitting electrical energy. US patent 1,119,732 (1914); https://patents.google.com/patent/US1119732

2. Gutruf, P. et al. Nat. Electron. 1, 652-660 (2018).

3. Boyvat, M., Koh, J.-S. \& Wood, R. J. Sci. Robot. 2, eaan1544 (2017).

4. Tian, X. et al. Nat. Electron. 2, 243-251 (2019).

5. Kurs, A. et al. Science 317, 83-86 (2007).

6. Lerosey, G. Nature 546, 354-355 (2017).

7. Assawaworrarit, S., Yu, X. \& Fan, S. Nature 546, 387-390 (2017). 